\section{Twin nasal cannula for administration of continuous positive airway pressure to newborn infants}

Endotracheal intubation with continuous positive airway pressure (CPAP) has been used successfully in infants with severe respiratory distress syndrome (RDS) (Gregory et al., 1971; De Lemos et al., 1972). The beneficial effects of CPAP are reflected by an increase in arterial $\mathrm{O}_{2}$ tension, a decrease in right to left shunt, and a reduction in the overall mortality (Gregory et al., 1971).

The most frequent complications of this treatment are related to the prolonged endotracheal intubation (De Lemos et al., 1972). To prevent these complications, new methods have recently been proposed that make use of different devices such as a face mask (Harris, 1972; Shannon et al., 1972), a head box (Gregory et al., 1971), or a plastic bag around the head (Barrie, 1972). All these devices, however, have some drawbacks. High flows of inspired gas are usually needed in order to maintain the desired pressure in spite of leaks and this creates problems of thermoregulation and noise level. Furthermore, the access to the baby's face for suctioning or feeding is often limited.

During the past 2 years we have used with good results a twin nasal cannula for delivering CPAP to newborn infants with RDS (Agostino et al., 1973). Different kinds of cannula have been tried and from the experience gained a final prototype has been designed which is presently used in our unit and is described here.

\section{Methods}

A schematic drawing of the device is shown in the Fig. The twin cannula was originally derived from a Portex Jackson-Rees nasotracheal tube having two short cannulae instead of a long one ${ }^{\star}$. A thin silastic tubing is glued around each tip of the polyvinylchloride cannulae. $\dagger$ The cannulae come in different sizes and should be introduced tightly into the infants nares in order to obtain a good fitting. Size 3 cannulae with silastic

\footnotetext{
«The twin nasal cannula was built for us by Portex Ltd., Hythe, Kent.

†Silastic Medical Grade Tubings (Cat. No. 602-305 and 601-335) and Silastic Medical Adhesive type A (Cat. No. 891) by Dow Corning were used.
}

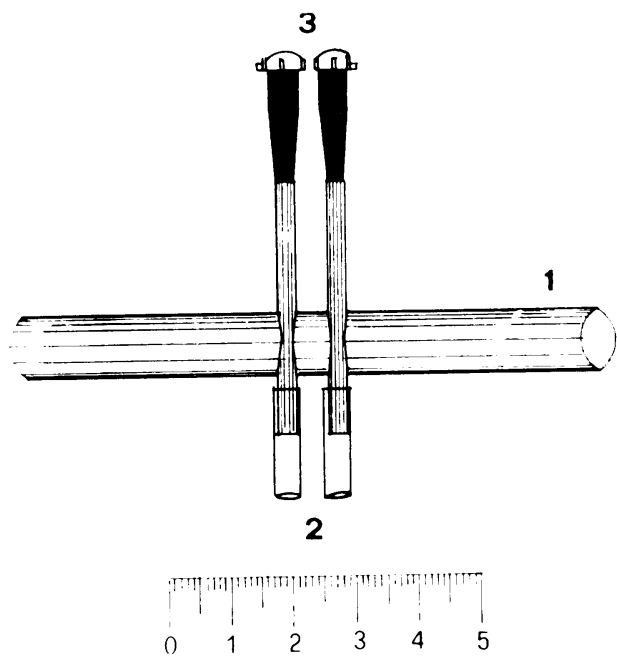

FIG.-Schematic representation of the twin nose cannula. The larger cross cannula (1) is connected to the continuous positive airway pressure (CPAP) circuit. The silastic tips (2) are introduced into the infant's nares. The stoppers (3) can be removed for nasopharyngeal suction or for measurements of inspired oxygen concentrations.

tubings of $4.65 \mathrm{~mm}$ O.D. are usually adequate for infants with a birthweight $>1700 \mathrm{~g}$; while size 2.5 cannulae with silastic tubings of $3 \cdot 18 \mathrm{~mm}$ O.D. can be used for smaller infants.

The silastic layer is important since we noticed that the use of plain polyvinylchloride cannulae was occasionally associated with necrotic lesions of the nasal septum. The length of the cannulae is equally important. Longer nasopharyngeal cannulae with tips extending beyond the choanal openings, similar to those recently described by Novogroder et al. (1973), were more difficult to insert and less well tolerated by the infants; they also tended to irritate the mucosa with the production of mucopurulent or membranous secretions. None of the above-mentioned complications was encountered with the new cannula described here. As shown in the Fig., the twin nasal cannula retains the advantages of the Jackson-Rees nasotracheal tube in that it can be easily fixed to the infant's head and suction can be applied without disturbing the baby after removing the stoppers at the external ends.

When delivering CPAP we connect the cannula to a circuit similar to that described by Gregory et al. (1971), with a few modifications. One of the features of our system is that the humidified gas mixture, flowing through the device at 4 to 61 ./minute, is allowed to 
bubble out through an exhaust tube immersed in a cylinder filled with water. In this way the pressure in the system can be finely regulated by varying the depth of the tubing under water, while at the same time the bubbling of gas in the cylinder provides a continuous monitoring for the possible presence of excessive leaks through the mouth. The circuit is completed by an rubber anaesthetic bag that serves as a buffer to minimize pressure swings due to breathing and/or bubbling, and by a graduated tube immersed by $30 \mathrm{~cm}$ into the cylinder that serves as an inverted manometer for reading the pressure in the circuit and as an emergency blow off valve.

All infants treated with CPAP have an orogastric tube in place, but we found that gastric distension is seldom a problem even with pressures up to 10 to $13 \mathrm{~cm} \mathrm{H}_{2} \mathrm{O}$. It is also usually unnecessary to tape the mouth since leaks through it are generally absent or tolerable.

\section{Results and discussion}

Since the introduction of the nasal cannulae we have admitted to our unit 33 premature infants with severe RDS requiring assisted respiration. 11 of these infants could be treated solely with the nasal cannula, while in 15 of them the nasal cannula was the prevailing form of treatment associated with CPAP by endotracheal intubation and/or intermittent positive pressure ventilation (IPPV). The remaining 7 infants required constant endotracheal intubation, either because they did not tolerate the cannula ( 2 infants) or because they were treated with IPPV, with or without positive end expiratory pressure.

Death occurred in $11(33 \%)$ patients of the whole series, and in $6(23 \%)$ of the 26 patients given only or predominantly nasal CPAP. Death was associated in all these cases with severe complications or other conditions, such as pneumonia in 5, sudden hypoventilation with intracranial haemorrhage 4, and pneumothorax 2 . Significant residual lung disease was not seen in survivors. The 33 infants required a total of 2417 hours of assisted respiration: 1105 hours $(46 \%$ of the total) were given by nasal cannula. Most patients required a CPAP of $+10 \mathrm{~cm} \mathrm{H}_{2} \mathrm{O}$ at the peak of their disease, but in some cases pressures up to $15 \mathrm{~cm} \mathrm{H}_{2} \mathrm{O}$ were given via the nasal cannula.

The Table shows the arterial oxygen tension $\left(\mathrm{Po}_{2}\right)$ measured in 3 premature infants who were initially treated with CPAP via an endotracheal tube, and in whom the tube was removed and CPAP reapplied with the twin nasal cannula, without changing the inspired $\mathrm{O}_{2}$ concentration and the positive pressure in the system. In all 3 infants the arterial $\mathrm{Po}_{2}$ with the nose cannula was remarkably similar to the $\mathrm{Po}_{2}$ obtained with the endoctracheal tube, lending
TABLE

Arterial oxygen tensions $\left(\mathrm{Po}_{2}\right)$ in 3 infants with $\mathrm{RDS}$ treated with CPAP applied by endotracheal intubation $(E I)$ and by nose cannula (NC)

\begin{tabular}{l|r|r|r|r|r|r|r}
\hline \multicolumn{1}{c|}{ Infants } & \multicolumn{2}{|c|}{1} & \multicolumn{2}{|c|}{2} & \multicolumn{2}{|c}{3} \\
\hline & EI & NC & EI & NC & EI & NC \\
\hline Inspired $\mathrm{O}_{2}(\%)$ & 95 & 95 & 80 & 80 & 98 & 98 \\
CPAP $\left(\mathrm{cm} \mathrm{H}_{2} \mathrm{O}\right)$ & 7 & 7 & 10 & 10 & 8 & 8 \\
Arterial $\mathrm{PO}_{2}$ (mmHg) & 65 & 67 & 120 & 118 & 130 & 130 \\
\hline
\end{tabular}

RDS, respiratory distress syndrome; CPAP, continuous positive airway pressure.

further support for the adequacy of our device for delivering CPAP.

The efficacy of a twin nose cannula for delivering CPAP to infants with RDS has been recently confirmed by Kattwinkel et al. (1973), who reported excellent results with the use of a silastic device similar to ours.

A nose cannula, therefore, provides a valid alternative to endotracheal intubation for the application of CPAP in infants with RDS. The method is simple to use and inexpensive, it is remarkably free from serious complications and allows avoidance of endotracheal intubation in many infants, and shortening of the period of intubation in most of the remaining cases.

\section{Summary}

Continuous positive airway pressure was applied in preterm infants by means of a silastic nasal cannula. This method of treatment is simple, inexpensive, and free from complications and either avoids or shortens endotracheal intubation in many infants with respiratory distress.

Supported in part by grants 72.00261 .43 and 72.00273.43 of the National Research Council (C.N.R.).

\section{REFERENCES}

Agostino, R., Orzalesi, M., Nodari, S., Mendicini, M., Conca, L., Savignoni, P. G., Picece-Bucci, S., Calliumi, G., and Bucci, G. (1973). Continuous positive airway pressure by nasal cannula in the respiratory distress syndrome of the newborn. (Abst.) Pediatric Research, 7, 50.

Barrie, H. (1972). Simple method of applying continuous positive airway pressure in respiratory-distress syndrome. Lancet, 1, 776.

De Lemos, R. A., Mac Laughlin, G. W., Diserens, H. W., and Kirby, R. R. (1972). Assisted ventilation in the treatment of hyaline membrane disease: the use of CPAP with or without assisted ventilation utilizing a single ventilator system. (Abst.) Pediatric Research, 6, 406.

Gregory, G. A., Kitterman, J. A., Phibbs, R. H., Tooley, W. H., and Hamilton, W. K. (1971). Treatment of the idiopathic respiratory-distress syndrome with continuous positive airway pressure. New England fournal of Medicine, 284, 1333.

Harris, T. R. (1972). Continuous positive airway pressure applied by face-mask. (Abst.) Pediatric Research, 6, 410. 
Kattwinkel, J., Fanaroff, A., Cha, C., Fleming, D., Sosa, R., Crumrine, R., and Klaus, M. (1973). Controlled trial of continuous positive airway pressure (CPAP) in RDS and a simplified application by the nasal route. (Abst.) Pediatric Research, 7, 396.

Novogroder, M., MacKuanying, N., Eidelman, A. I., and Gartner, L. M. (1973). Nasopharyngeal ventilation in respiratory distress syndrome. Fournal of Pediatrics, 82, 1059.

Shannon, D. C., Lusser, M., Goldbatt, A., and Bunnel, J. B. (1972). The cyanotic infant. Heart disease or lung disease. New England Journal of Medicine, 287, 951.

G. Caliumi-Pellegrini, R. Agostino, M. Orzalesi, S. Nodari, G. Marzetti, P. G. Savignoni, and G. BUCCI*

Institute of Paediatrics, Neonatal Division, University of Rome Medical School, Rome, Italy.

*Correspondence to Dr. G. Bucci, Clinica Pediatrica Università, Viale Regina Elena 324, 00161 Roma, Italy.

\section{Phrenic nerve palsy treated by continuous positive pressure breathing by nasal cannula}

Early respiratory failure and death may occur in newborns with phrenic nerve palsy (Richard et al., 1957). We report 2 cases where newborn infants with respiratory failure due to this condition were successfully managed by continuous positive airway pressure (CPAP) breathing administered by nasal cannula.

\section{Methods}

Acid-base status and blood gases were determined on samples from the radial artery or the descending aorta by a micro-Astrup apparatus and a Clark-type microelectrode (Orzalesi et al., 1967). CPAP was administered by a twin nasal cannula and an apparatus as described by Caliumi-Pellegrini et al. (1974). The central venous pressure was measured with an umbilical venous catheter and a Hewlett Packard 1280C pressure transducer and $7819 \mathrm{~A}$ pressure monitor, visualized by a 7803A monitor scope. The tip of the catheter was located at the right atrial inlet by an internal electrocardioscopical method (Nodari, Agostino, and Bucci, 1973).

\section{Case reports}

Case 1. A male, born at 35 weeks' gestation in a small community hospital. The mother had a small pelvis, and the presentation was a breech dorsal posterior. The delivery was by a midwife, who had to exert considerable traction to extract the baby. At birth the infant was cyanosed but breathing began spontaneously, and he remained normal for about 30 minutes after delivery. Thereafter, respiratory movements became increasingly distressed.

He was transferred to this unit at 6 hours of age, with a weight of $2640 \mathrm{~g}$, severe respiratory distress, and cyanosis in $100 \%$ oxygen. Deep inspiratory excursions of the chest, with marked withdrawal of the upper abdominal quadrants bilaterally, were conspicuous. Fine rales were heard over both lungs. The heart sounds, a rate of $150 / \mathrm{min}$, were not displaced. The abdomen was scaphoid, and the liver edge was palpable at the right costal margin. There was decreased muscular tone and movement of the upper limbs, a weak Moro response, absent suction and rooting reflexes, and a weak palmar and plantar grasp. At 7 hours of age, a chest $x$-ray showed marked decrease of expansion of lung fields, right diaphragm at the 6th rib and left diaphragm at the 7th intercostal space, atelectasis at both upper lobes, slight shift of the mediastinum to the left. This suggested phrenic nerve palsy (complete on the right side, and possibly present to some degree also on the left side).

An umbilical venous catheter was inserted up to the right atrial inlet. An umbilical arterial catheter was also introduced. After $22 \mathrm{mEq} \mathrm{NaHCO}_{3}$ had been administered because of severe combined acidosis, the acid-base status was normal but the arterial $\mathrm{Po}_{2}$ was relatively low in $100 \%$ oxygen (Table I, at 8 hours).

TABLE I

Case 1. Acid-base and oxygen determinations on arterial (umbilical) blood (see text)

\begin{tabular}{|c|c|c|c|c|c|}
\hline Age (hr) & 8 & 11 & 44 & $44 \cdot 5$ & 45 \\
\hline $\begin{array}{l}\mathrm{F}_{\mathrm{r}} \mathrm{O}_{2} \\
\mathrm{CPAP}\left(\mathrm{cm} \mathrm{H} \mathrm{H}_{2} \mathrm{O}\right) \\
p \mathrm{H} \\
\text { Base excess } \\
\quad(\mathrm{mEq} / \mathrm{l}) \\
\mathrm{PCO}_{2}(\mathrm{mmHg}) \\
\mathrm{PO}_{2}(\mathrm{mmHg}) \\
\mathrm{SaO}_{2}(\%)\end{array}$ & $\begin{array}{c}1 \cdot 0 \\
\overline{7 \cdot 34} \\
0 \\
46 \\
65 \\
92 \cdot 8\end{array}$ & $\begin{array}{c}1 \cdot 0 \\
+5 \\
7 \cdot 34 \\
+1 \\
48 \\
208 \\
100\end{array}$ & $\begin{array}{c}0 \cdot 4 \\
+5 \\
7 \cdot 40 \\
+5 \\
58 \\
126 \\
98 \cdot 5\end{array}$ & $\begin{array}{c}0 \cdot 4 \\
+3 \\
7 \cdot 47 \\
+5 \\
42 \\
124 \\
99 \cdot 5\end{array}$ & $\begin{array}{c}0.4 \\
+3 \\
7 \cdot 44 \\
+5 \\
48 \\
64 \\
94 \cdot 6\end{array}$ \\
\hline
\end{tabular}

The mean central venous pressure was $+8 /+9 \mathrm{~cm} \mathrm{H}_{2} \mathrm{O}$, with no respiratory oscillations seen on the oscilloscope even during the deepest inspirations. In view of the persistence of severe respiratory distress, CPAP breathing was started with the pressure of $5 \mathrm{~cm} \mathrm{H}_{2} \mathrm{O}$, and was immediately followed by improvement. Thoracic inspiratory efforts and abdominal inspiratory withdrawing decreased immediately. The rales cleared gradually over the next few hours. An arterial sample taken 30 minutes after the onset of CPAP breathing (Table I, at 11 hours) showed no acid-base changes and conspicuously increased $\mathrm{PO}_{2}$ with respect to the previous findings. The CVP rose to $+12 /+14 \mathrm{~cm} \mathrm{H}_{2} \mathrm{O}$ for about 30 minutes, but then returned to previous levels. A chest $x$-ray, taken at 26 hours of age, showed much improved lung expansion, and disappearance of pulmonary opacities and of the mediastinal shift.

Over the first 24 hours after the onset of CPAP breathing there was a gradual improvement of arterial $\mathrm{PO}_{2}$ and $p \mathrm{H}$ levels, and it was possible to keep $\mathrm{PaO}_{2}$ levels between 70 and $130 \mathrm{mmHg}$ by gradually decreasing the $\mathrm{F}_{2}$ to $0 \cdot 40-0 \cdot 45$. In the first week of life, attempts to discontinue or even to decrease the CPAP below $+5 \mathrm{~cm}$ $\mathrm{H}_{2} \mathrm{O}$ were immediately followed by a marked increase of respiratory distress. Arterial acid-base and oxygen 